\title{
Emergencias y urgencias obstétricas en el Hospital Central Militar (I): nuestra visión y el horizonte epidemiológico
}

\author{
Obstetric emergencies and non-emergencies at Central Military Hospital (I): Our vision \\ and the epidemiologic horizon
}

\begin{abstract}
Luis M. García-Núñez ${ }^{1 *}$, Juan A. Ramos-Martínez², Irving I. Morales-Pogoda³, Martín Fuentes-Durán Edgar F. Hernández-García ${ }^{5}$ y Raúl García-Ramírez

${ }^{1}$ Área de Medicina Crítica; ${ }^{2}$ Departamento de Urgencias; ${ }^{3}$ Departamento de Medicina Intensiva, Área de Medicina Crítica. Hospital Central Militar, SEDENA; ${ }^{4}$ Cirugía General, Escuela Militar de Graduados de Sanidad, Universidad del Ejército y Fuerza Aérea; ${ }^{5}$ Subsección de Cirugía de Trauma, Departamento de Cirugía; ${ }^{6}$ Subdirección General. Hospital Central Militar, SEDENA. Ciudad de México, México
\end{abstract}

\begin{abstract}
RESUMEN
Introducción: La morbimortalidad materna posee un significativo impacto en la salud pública nacional, siendo la atención médica de las emergencias obstétricas (EO) y urgencias obstétricas (UO) de capital importancia. Método: Análisis descriptivo y epidemiológico de EO/UO en un escalón militar de tercer nivel. Resultados: Durante 34 meses se abordaron en el departamento de urgencias 48 pacientes (1.4 admisiones/mes). La edad media fue de $29 \pm 3$ años (rango: 17-41). Ocho pacientes (17\%) se consideraron EO y 40 (83\%) UO. El 58\% $(n=28)$ de las pacientes se admitieron a la institución; el 32\% ( $n=9)$ se manejaron médicamente y el $68 \%(n=19)$ con tratamiento quirúrgico. La causa más importante de admisión fue la hemorragia posoperatoria (22\%; $n=6)$. Las intervenciones quirúrgicas más frecuentes fueron maniobras de hemostasia quirúrgica (31.5\%; $n=6)$. El $82 \%(n=23)$ de las admisiones requirieron manejo en la unidad de medicina intensiva (UMI), con una estancia media de $6.4 \pm 4.9$ días (rango: 2-21). El 35\% $(n=8)$ requirieron ventilación mecánica. La puntuación media APACHE II en la UMI fue de $19.4 \pm 8.4$, y la probabilidad predicha de muerte fue del $35.5 \%$. La tasa global de morbilidad fue del $27 \%$ (1.8 complicaciones/paciente). La tasa de mortalidad global fue del 6.2\%; la mortalidad específica para pacientes embarazadas del $0 \%(n=0)$ y para pacientes puérperas del $12.5 \%(n=3)$. La tasa de mortalidad en la UMI fue del $4.3 \%(n=1)$. Conclusiones: El Hospital Central Militar ha delineado y definido diversos procedimientos para abatir la morbimortalidad maternas. La correcta práctica de estos procedimientos contribuirá a alcanzar los objetivos institucionales deseados.
\end{abstract}

PALABRAS CLAVE: Mortalidad materna. Emergencia obstétrica. Morbilidad materna.

\section{Abstract}

Background: Maternal morbidity and mortality pose a significant impact on national public health, being medical attention of obstetric emergencies (OE) and non-emergencies (ONE) of capital importance. Methods: Descriptive and epidemiologic analysis of OE/ONE at a 3rd level military echelon. Results: During a 34-months span, 48 patients were approached at the emergency department (1.4 admissions/month). Mean age: $29 \pm 3$ years (17-41). Eight patients (17\%) were considered OE and 40 (83\%) ONE. Fifty-eight percent $(n=28)$ of patients were admitted to our institution; $32 \%(n=9)$ were managed under non-surgically basis and 68\% $(n=19)$ underwent surgical therapy. Most important cause of admission: postoperative hemorrhage (22\%; $n=6)$. Most frequent operative interventions: surgical hemostasis maneuvers $(31.5 \% ; n=6)$. Eighty-two percent $(n=23)$ of admissions required management at intensive care unit (ICU), with mean length of stay of $6.4 \pm 4.9$ days (2-21). Thirty-five percent $(n=8)$ required mechanical ventilation. Mean score of APACHE II at ICU: $19.4 \pm 8.4$; predicted probability

\author{
Correspondencia: \\ *Luis Manuel García-Núñez \\ Av. Ejército Nacional y Blvd. \\ Manuel Ávila Camacho, s/n \\ Col. Lomas de Sotelo, Del. Miguel Hidalgo \\ C.P. 11200, Ciudad de México, México \\ E-mail: Imgarcian@hotmail.com
}

Fecha de recepción: 15-01-2017

Fecha de aceptación: 08-04-2018

DOI://dx.doi.org/10.24875/CIRU.M18000025
Cir Cir. 2018;86:161-168

Contents available at PubMed www.cirugiaycirujanos.com 
of death: $35.5 \%$. Global morbidity rate: $27 \%$ (1.8 complications/patient). Global mortality rate: $6.2 \%$; specific mortality for pregnant patients $0 \%(n=0)$ and for post-partum patients $12.5 \%(n=3)$. Mortality rate at ICU: $4.3 \%(n=1)$. Conclusions: Central Military Hospital has delineated and defined several procedures to decrease maternal morbidity and mortality. Appropriate practice of these procedures contributes to reach the desired institutional objectives.

KEY WORDS: Maternal mortality. Obstetric emergency. Maternal morbidity.

\section{Introducción}

La mortalidad materna (entendida como la muerte de una mujer durante el embarazo o dentro de los 42 días siguientes a su término, independientemente de la duración y el sitio del embarazo, debido a causas directamente relacionadas con el embarazo y sus efectos, condiciones obstétricas, o bien enfermedades preexistentes o evolutivas durante el embarazo, no debidas a causas obstétricas, pero sí agravadas por el estado de gestación) es un índice sensible de la emergencia nacional, pues su elevada tasa traduce inequidad, falta de acceso a los servicios de salud y deficiente calidad en la atención obstétrica'. En Méxi$\mathrm{co}$, desgraciadamente, prevalece como un grave problema epidemiológico y constituye un reto enorme para las autoridades sanitarias públicas y privadas ${ }^{2}$.

Según las cifras de la Organización Mundial de la Salud, en 2015 se registraron en el mundo cerca de 303,000 muertes por complicaciones del embarazo o del puerperio. La mayor parte de los decesos ocurrieron en países con bajo desarrollo sociopolítico y se relacionan, sin duda, con bajos ingresos económicos. En México, la estadística más reciente y confiable data de 2014 (Secretaría de Salud) y es verdaderamente alarmante: en ese año se computaron 872 muertes maternas (2.3 al día), con un $71 \%$ de ellas en solo 12 entidades federativas. Sin embargo, es notable que la razón de muerte materna (RMM) ha disminuido de 1990 a la fecha (RMM 89 versus RMM 38.9), lo que refleja el cumplimiento de los Objetivos de Desarrollo Sostenible definidos por el Gobierno Federal, con una tendencia general de reducción en la mortalidad materna en la nación ${ }^{1-4}$.

Las políticas administrativas y asistenciales que desde 2012 prevalecen y son observadas en el Hospital Central Militar dejan ver con claridad la gran importancia que para la institución revisten la mortalidad y la morbilidad maternas. Debido a ello, el área de medicina crítica y sus departamentos subalternos desarrollaron la primera de tres fases de análisis de la atención de emergencias y urgencias obstétricas en la institución (fase epidemiológica, propuesta de protocolos y diseño de protocolos); en esta primera fase se delineará un perfil epidemiológico de las pacientes que arriben con la definición operativa de «emergencia obstétrica» (EO) o «urgencia obstétrica» (UO) (Tabla 1) ${ }^{1}$.

Se aplicó la estadística descriptiva y analítica para identificar los factores de riesgo significativos para el aumento de la morbimortalidad, determinando la incidencia de la enfermedad en nuestra población. Se estudió el pronóstico de la enfermedad, analizando los indicadores para medir la gravedad utilizados en las diferentes áreas de abordaje, y el uso de estos para definir el tratamiento definitivo de las pacientes, evaluando la eficacia del tratamiento médico y quirúrgico aplicado en nuestra institución con un enfoque simple y estandarizado para generar, analizar y difundir la información, y poder comparar nuestros resultados con lo que apunta la literatura mundial, hechos de elevada importancia para formular políticas de salud ${ }^{1-4 .}$

Finalmente, el estudio intenta contribuir al diseño y la validación de protocolos de abordaje médico-quirúrgico en pacientes que cursan con EO y UO, lo que permitirá mejorar el empleo de recursos humanos, materiales y administrativos, identificando el momento oportuno para aplicar cada uno de ellos; y con ello optimizar los índices de sobrevida y funcionalidad, así como disminuir la tasa de complicaciones invalidantes, elevando de esta forma la calidad de la atención médica y promoviendo el bienestar social ${ }^{1-4}$.

\section{Método}

Se efectuó una revisión del archivo electrónico y físico del Hospital Central Militar, en un tiempo de 34 meses (de enero de 2015 a octubre de 2017), con objeto de detectar los expedientes de las pacientes que cumplieran con las definiciones operativas de EO y UO, obteniendo del total de los pacientes que se admitieron por el departamento de urgencias de 
Tabla 1. Definiciones operativas para el Hospital Central Militar

\begin{tabular}{|c|c|}
\hline Variable & Definición operativa \\
\hline Embarazo & Periodo comprendido desde la concepción hasta la expulsión o extracción del feto y sus anexos. \\
\hline Puerperio & $\begin{array}{l}\text { Periodo que sigue a la expulsión del producto de la gestación, en el cual revierten los cambios } \\
\text { anatomofisiológicos propios del embarazo. Tiene una duración de } 6 \text { semanas o } 42 \text { días. y se divide en: } \\
\text { Inmediato: comprende las primeras } 24 \text { horas después del parto. } \\
\text { Mediato: periodo que abarca del segundo al séptimo días después del parto. } \\
\text { Tardío: periodo que incluye del día } 8 \text { al } 42 \text { después del parto. }\end{array}$ \\
\hline Emergencia obstétrica (EO) & $\begin{array}{l}\text { Estado que pone en peligro la vida de la mujer o del producto de la gestación durante la etapa } \\
\text { grávido-puerperal, que requiere atención médica o quirúrgica inmediata por personal médico calificado }\end{array}$ \\
\hline Urgencia obstétrica (UO) & $\begin{array}{l}\text { Complicación médica durante la gestación, el parto o el puerperio, que incrementa el riesgo de morbilidad o } \\
\text { mortalidad materna y perinatal. }\end{array}$ \\
\hline Triage obstétrico & $\begin{array}{l}\text { Protocolo de atención de primer contacto en EO, que tiene como propósito clasificar la situación de gravedad } \\
\text { de las pacientes y precisar la acción necesaria para preservar la vida del binomio o la viabilidad de un órgano } \\
\text { dentro de un lapso terapéutico. El sistema se ha adaptado para emplearse en el periodo perinatal (embarazo, } \\
\text { parto y puerperio) y en cada contacto de la paciente con el personal de salud. Cuando una paciente es } \\
\text { identificada con alguna complicación o emergencia, se enlaza y se activa la ruta crítica para la vigilancia del } \\
\text { embarazo ( «Código máter»). }\end{array}$ \\
\hline Código máter & $\begin{array}{l}\text { Activación de un mecanismo de llamado al personal del equipo de respuesta inmediata obstétrica para atender } \\
\text { una emergencia y salvar la vida de la madre y del producto de la gestación. }\end{array}$ \\
\hline
\end{tabular}

Adaptada de: Subsecretaría de Prevención y Promoción de la Salud. Lineamiento Técnico 2016.

nuestra institución $(\mathrm{N}=1190)$ a $48(4 \%)$ que reunieron los criterios para ser incluidas en este análisis.

Debido a que los recursos humanos y materiales para la atención ginecoobstétrica y pediátrica se encuentran fuera de nuestro hospital (Hospital Militar de Especialidades de la Mujer y Neonatología, HMEMYN), todas las pacientes de nuestra población (militares en el activo y derechohabientes que radican en el área metropolitana) durante la gestación, el parto y el puerperio se tratan inicialmente en ese escalón médico, y se refieren a nuestro hospital (Hospital Central Militar) las pacientes con EO y UO que requieren continuar con su abordaje por el Equipo Multidisciplinario de Respuesta Inmediata Obstétrica (EMRIO), conformado por personal de salud experto de diferentes especialidades con las que no cuenta el HMEMYN.

Ante el arribo de una paciente de esta naturaleza, se llevó a cabo una categorización de su estado clínico con la escala de triage obstétrico ${ }^{1}$, a fin de asignar algún grado de prioridad a su atención. En los casos categorizados como "Código rojo» se activó el protocolo institucional denominado "Código máter» para alertar, conformar y accionar el EMRIO, integrado por médicos especialistas del área médica y quirúrgica, así como por residentes de la especialidad, enfermeras y personal de apoyo administrativo, según lo establecido en el lineamiento técnico "Triage obstétrico, código máter y equipo de respuesta inmediata obstétrica» (Secretaría de Salud, 2016) ${ }^{1,3,5}$.

Fue necesario para la institución implementar protocolos administrativos para atraer de forma expedita (dentro de los siguientes 30 minutos a su llamado) la presencia de estos médicos especialistas externos en el departamento de urgencias, al ser aplicable. Por otro lado, las pacientes en «Código amarillo» 0 «Código verde" se manejaron por su médico tratante, con los procedimientos correspondientes estatuidos en el Manual de guías clínicas del departamento de urgencias. Cuando fue requerido, se accionó el protocolo denominado "Código binomio", que se emplea para la atención de pacientes embarazadas que arriban en fase de expulsión.

Del expediente conformado por el proceso de atención médica se obtuvieron los valores correspondientes a variables demográficas, la categoría en el triage obstétrico, la edad gestacional (semanas de embarazo), la fase del puerperio, las intervenciones realizadas y los índices pronósticos (complicaciones específicas y mortalidad). Debido a la naturaleza indagatoria de la investigación, los datos de las variables antes señaladas se sujetaron a un análisis estadístico con medidas de centralización y dispersión para el reporte descriptivo, además de emplear la frecuencia relativa de ocurrencia para estimar las proporciones correspondientes a las características de la población de estudio. 


\section{Resultados}

Durante el periodo de estudio se admitieron por el departamento de urgencias de nuestra institución 48 pacientes que reunieron los criterios operativos para ser incluidas en este análisis, con una media de 1.4 admisiones por mes. El promedio de edad fue de 29 \pm 3 años (rango: 17-41). De ellas, ocho pacientes fueron consideradas emergencias obstétricas (EO, «Código rojo" del triage obstétrico), representando el 17\% de los casos, y 40 pacientes (83\%) se categorizaron como urgencias obstétricas (UO, "Código amarillo» y «Código verde» del triage obstétrico) (Fig. 1)1,2.

En nuestra serie, es notable que la causa más importante de admisión al departamento de urgencias fue la hemorragia posoperatoria $(n=6 ; 12.5 \%)$, casos categorizados como genuinas EO y en «Código rojo» en la escala de triage obstétrico, seguida por la amenaza de aborto y los trastornos hipertensivos del embarazo no graves ( $n=5 ; 10.5 \%$ cada una). El resto de las causa de admisión se describen en la tabla 2.

Como dato especialmente importante para la implementación del análisis SWOT y de las acciones de mejora consecuentes en nuestra institución, que se desarrollarán en las fases II y III del presente análisis, es trascendental señalar que 26 pacientes (54\%) fueron referidas de escalones sanitarios militares externos o de servicios de salud no institucionales, y 22 pacientes (46\%) arribaron directamente al hospital, ya sea procedentes de su domicilio o de la vía pública (Fig. 2).

Veinticuatro pacientes arribaron embarazadas $(50 \%)$, con una edad gestacional promedio de $16 \pm 4$ semanas (rango: 7-39) (Fig. 3). De ellas, cinco (21\%) se presentaron en fase de expulsión del producto en el departamento de urgencias. Tras la atención del trabajo de parto, estas pacientes fueron trasladadas junto con el producto, en buenas condiciones clínicas y de forma expedita, al HMEMYN, para dar seguimiento por las especialidades correspondientes. Es notable el caso de una paciente que expulsó un producto óbito de 19 semanas inmediatamente a su llegada al servicio, atendiendo con rapidez su estado físico, pues arribó con estado de choque hipovolémi$\mathrm{co}$, siendo posteriormente trasladada al hospital de la especialidad.

Veinticuatro pacientes (50\%) arribaron cursando su puerperio (Fig. 3). Doce pacientes se presentaron en su puerperio fisiológico y un igual número de casos cursando su puerperio quirúrgico (50\% para ambos casos). La duración media del puerperio fue de $2 \pm$
Tabla 2. Causas de admisión al departamento de urgencias ( $n=48)$

\begin{tabular}{|c|c|}
\hline Causa & $\mathrm{n} / \mathrm{N}(\%)$ \\
\hline Hemorragia posoperatoria & $6 / 48(12.5)$ \\
\hline Amenaza de aborto & $5 / 48(10.5)$ \\
\hline $\begin{array}{l}\text { Trastornos hipertensivos del embarazo } \\
\text { (no graves) }\end{array}$ & $5 / 48(10.5)$ \\
\hline $\begin{array}{l}\text { Trastornos hipertensivos del embarazo (graves) y sus } \\
\text { complicaciones }\end{array}$ & $5 / 48(10.5)$ \\
\hline Infección urinaria en el embarazo & $4 / 48(8.3)$ \\
\hline Traumatismos de baja transmisión de energía & $3 / 48(6.3)$ \\
\hline Embarazo ectópico (roto) & $3 / 48(6.3)$ \\
\hline Embarazo ectópico (no roto) & $2 / 48(4.2)$ \\
\hline $\begin{array}{l}\text { Enfermedad litiásica de la vía biliar y sus } \\
\text { complicaciones (excluyendo pancreatitis aguda de } \\
\text { origen biliar) }\end{array}$ & 2/48 (4.2) \\
\hline Pancreatitis aguda de diversas etiologías & $2 / 48(4.2)$ \\
\hline Sepsis grave no quirúrgica de origen variado & $2 / 48(4.2)$ \\
\hline Sepsis posquirúrgica & 2/48 (4.2) \\
\hline Apendicitis aguda & $1 / 48(2.1)$ \\
\hline Fibrosis pulmonar idiopática & $1 / 48(2.1)$ \\
\hline Perforación gastrointestinal & $1 / 48(2.1)$ \\
\hline Tromboembolia pulmonar & $1 / 48(2.1)$ \\
\hline Falla orgánica & $1 / 48(2.1)$ \\
\hline Evento cerebrovascular & $1 / 48(2.1)$ \\
\hline $\begin{array}{l}\text { Agudizaciones de enfermedades crónicas durante el } \\
\text { embarazo o el puerperio }\end{array}$ & $1 / 48(2.1)$ \\
\hline
\end{tabular}

Fuente: Control de Trabajo Social y Archivos del Departamento de Urgencias, Hospital Central Militar, 2017; Archivo del Hospital Central Militar, 2017.

0.7 días (rango: 0-4) para las cursantes del puerperio fisiológico y de $0.9 \pm 1.9$ días (rango: $0-10$ ) para las de puerperio quirúrgico.

Veinte pacientes (42\%), incluyendo las embarazadas $(n=5)$, fueron atendidas y estabilizadas en el departamento de urgencias para ser inmediatamente trasladadas al HMEMYN, ya sea por su médico tratante o tras la evaluación y el tratamiento por parte del ERMIO. Las causas más comunes de atención de estas pacientes en el departamento de urgencias fueron la amenaza de aborto y los trastornos hipertensivos no graves del embarazo (5/48 [10.5\%] cada uno); (Tabla 2). Sin embargo, algunas de ellas ( $n=28 ; 58 \%)$ tuvieron que permanecer en la institución para ser admitidas y sujetas a manejo médico-quirúrgico especializado. El diagnóstico que más frecuentemente motivó la admisión hospitalaria de las pacientes fue 


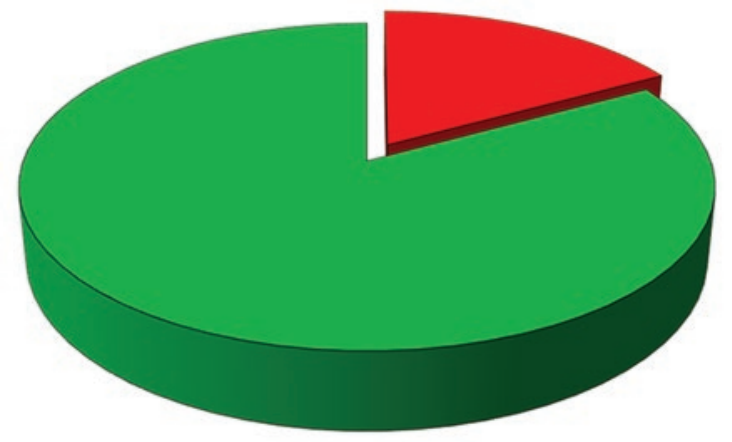

घEmergencias obstétricas ( $n=8 ; 17 \%)$ uUrgencias obstétricas $(n=40 ; 83 \%)$

Figura 1. Distribución de las emergencias y urgencias obstétricas ( $N=48)$. (Fuente: Archivo del Hospital Central Militar, 2017).

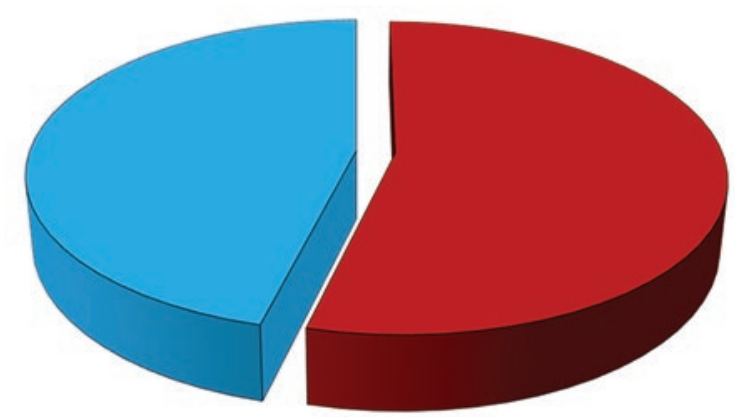

aPacientes referidas $(n=26 ; 54 \%)$ aPacientes de arribo directo $(n=22 ; 46 \%)$

Figura 2. Distribución de las pacientes referidas de otros servicios de salud y de arribo directo al departamento de urgencias $(N=48)$. (Fuente: Archivo del Hospital Central Militar, 2017).

la hemorragia posoperatoria $(n=6 ; 22 \%)$, seguido por los trastornos hipertensivos graves del embarazo y sus complicaciones $(n=5 ; 19 \%$, incluyendo tres casos de rotura hepática por síndrome HELLP [hemolysis, elevated liver enzimes, low platelets count]), y el embarazo ectópico roto $(n=3 ; 11 \%)$. El resto de las causas de admisión se exhibe en la tabla 3.

Con fundamento en lo anterior, se encontró que, de los 28 casos admitidos, nueve se manejaron de forma médica (32\%), mientras que la terapia quirúrgica fue requerida en 19 (68\%). Las intervenciones quirúrgicas que más comúnmente se instituyeron para el manejo de estos casos fueron las maniobras de hemostasia quirúrgica (ligadura vascular, electrocoagulación, empaquetamiento, aplicación de hemostáticos tópicos), necesarias en seis casos (31.5\%) de hemorragia posquirúrgica, seguidas por la laparotomía exploradora con control de la hemorragia hepática y para cohibir el sangrado procedente de un embarazo ectópico roto ( $n=3 ; 16 \%$ cada uno) (Tabla 4). Es importante señalar que la angiografía con angioembolización selectiva,

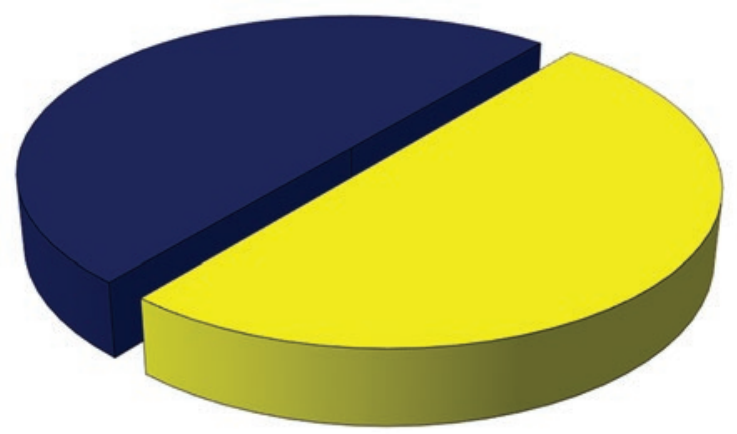

口Pacientes embarazadas ( $n=24 ; 50 \%)$ - Pacientes puérperas $(n=24 ; 50 \%)$

Figura 3. Distribución de las pacientes embarazadas y puérperas ( $N=48)$. (Fuente: Archivo del Hospital Central Militar, 2017).

un recurso de alta tecnología y disponibilidad permanente en nuestro hospital, fue realizado en cinco pacientes como adyuvante al control operatorio del sangrado.

Debido a sus condiciones de gravedad, el $82 \%$ (23/28) de las pacientes admitidas hospitalariamente requirieron manejo integral en la unidad de medicina intensiva (UMI). La estancia media de estas pacientes fue de $6.4 \pm 4.9$ días (rango: 2-21). El 35\% (8/23) de las pacientes admitidas en la UMI requirieron ventilación mecánica en algún momento de su estancia. Una paciente $(4 \%)$ con falla renal requirió terapia de reemplazo de la función renal. La puntuación media de arribo a la UMI en la escala APACHE II fue de $19.4 \pm$ 8.4 (rango: 7-37), lo cual refleja el estado fisiológico crítico de las pacientes manejadas en la mencionada unidad y su alta probabilidad predicha de muerte, correspondiendo en estos casos al $35.5 \%$.

Dentro de las complicaciones, la tasa global de morbilidad en nuestra serie alcanzó el 27\% (13/48), encontrando 24 complicaciones específicas para una media de 1.8 complicaciones específicas por paciente. Para efectos de este estudio, creemos que aquellas que revisten mayor importancia son las relacionadas con las complicaciones sépticas, la falla orgánica (principalmente respiratoria y renal), la coagulopatía, los fenómenos tromboembólicos y los accidentes neurológicos, por lo cual su estratificación se fundamentó en este razonamiento. Sobre esta base, la falla renal fue la complicación específica más frecuente, habiéndose presentado en el $46 \%$ (6/13) de las pacientes con complicaciones, seguida por la coagulopatía y la sepsis de origen pulmonar (38.5\%; 5/13). La distribución del resto de la morbilidad específica se describe en la tabla 5 .

La tasa de mortalidad global fue del $6.2 \%$. La mortalidad específica para el grupo de pacientes embarazadas 
Tabla 3. Causas (diagnósticos) de permanencia hospitalaria para admisión ( $n=28)$

\begin{tabular}{lc}
\hline Causa & n/n (\%) \\
\hline Hemorragia posoperatoria & $6 / 28(21)$ \\
Trastornos hipertensivos del embarazo (graves) y sus & $5 / 28(18)$ \\
complicaciones & \\
Embarazo ectópico roto & $3 / 28(11)$ \\
Enfermedad litiásica de la vía biliar y sus & $2 / 28(7)$ \\
complicaciones (excluyendo pancreatitis aguda de & \\
origen biliar) & \\
Pancreatitis aguda de diversas etiologías & $2 / 28(7)$ \\
Sepsis no quirúrgica de origen variado & $2 / 28(7)$ \\
Sepsis posquirúrgica & $2 / 28(7)$ \\
Apendicitis aguda & $1 / 28(3.5)$ \\
Fibrosis pulmonar idiopática & $1 / 28(3.5)$ \\
Perforación gastrointestinal & $1 / 28(3.5)$ \\
Tromboembolia pulmonar & $1 / 28(3.5)$ \\
Falla orgánica & $1 / 28(3.5)$ \\
Evento cerebrovascular & $1 / 28(3.5)$ \\
\hline Fuente: Archivo del Hospital Central Militar, 2017. &
\end{tabular}

Tabla 4. Estrategias operatorias para el tratamiento de pacientes sometidas a tratamiento quirúrgico $(n=19)$

\begin{tabular}{lc}
\hline Estrategia operatoria* & $\mathbf{n} / \mathbf{n}(\%)$ \\
\hline $\begin{array}{l}\text { Hemostasia quirúrgica (ligadura vascular, } \\
\text { electrocoagulación, empaquetamiento y aplicación } \\
\text { de hemostáticos tópicos) }\end{array}$ & $6 / 19(31.5)$ \\
$\begin{array}{ll}\text { Laparotomía exploradora con hepatostasia } \\
\text { Laparotomía exploradora para cohibir el sangrado }\end{array}$ & $3 / 19(16)$ \\
procedente de un embarazo ectópico roto & \\
$\begin{array}{l}\text { Limpieza quirúrgica (secuencial), control de fuente de } \\
\text { sepsis y manejo con abdomen abierto }\end{array}$ & $3 / 19(16)$ \\
Colecistectomía laparoscópica & $2 / 19(11)$ \\
Apendicectomía laparoscópica & $1 / 19(5)$ \\
$\begin{array}{l}\text { Toracotomía en el departamento de urgencias, con } \\
\text { manejo de tórax y abdomen abierto }\end{array}$ & $1 / 19(5)$ \\
\hline
\end{tabular}

*En cinco pacientes se empleó la angiografía con angioembolización selectiva como adyuvante al control operatorio del sangrado.

Fuente: Archivo del Hospital Central Militar, 2017.

fue del $0 \%(0 / 24)$, mientras que para el grupo de pacientes puérperas fue del $12.5 \%$ (3/24). Una paciente puérpera falleció en el departamento de urgencias por tromboembolia pulmonar masiva, mientras que otra, también cursando su puerperio, sucumbió en la unidad de hospitalización regular por neumonitis intersticial idiopática en etapa fibrótica. Una paciente con
Tabla 5. Distribución de la morbilidad específica $(n=13)$

\begin{tabular}{|c|c|}
\hline $\begin{array}{l}\text { Tipo de } \\
\text { complicación* }\end{array}$ & Complicación específica (n/n; \%) \\
\hline $\begin{array}{l}\text { Complicaciones } \\
\text { sépticas }\end{array}$ & $\begin{array}{l}\text { Sepsis pulmonar }(5 / 13 ; 38.5) \\
\text { Sepsis abdominal persistente }(3 / 13 ; 23)\end{array}$ \\
\hline Falla orgánica & $\begin{array}{l}\text { Falla renal }(6 / 13 ; 46) \\
\text { Falla hepática }(2 / 13 ; 15.5) \\
\text { Falla cardiaca }(1 / 13 ; 8)\end{array}$ \\
\hline Coagulopatía & $\begin{array}{l}\text { Coagulopatía asociada al embarazo o } \\
\text { puerperio }(5 / 13 ; 38.5)\end{array}$ \\
\hline $\begin{array}{l}\text { Accidentes } \\
\text { neurológicos }\end{array}$ & Evento cerebrovascular isquémico $(1 / 13 ; 8)$ \\
\hline Otros & Arritmia letal $(1 / 13 ; 8)$ \\
\hline \multicolumn{2}{|c|}{$\begin{array}{l}\text { ॠNo se encontraron complicaciones tromboembólicas, aunque este fenómeno fue la } \\
\text { causa de la muerte de una paciente puérpera en el departamento de urgencias } \\
\text { (Fuente: Archivo del Hospital Central Militar, 2017). } \\
\text { tuberculosis peritoneal y sepsis abdominal incontrolabl } \\
\text { falleció en la UMI, para una mortalidad específica er } \\
\text { esta unidad del } 4.3 \%(1 / 23) \text {. }\end{array}$} \\
\hline
\end{tabular}

\section{Discusión}

Por su repercusión en la economía nacional y en la moral y el crecimiento de la sociedad, la salud materna es un rubro de la atención sanitaria de máximo interés para cualquier país. En México, la incidencia y la prevalencia de las complicaciones y las muertes maternas han obligado a las autoridades gubernamentales y a las organizaciones no gubernamentales a instituir medidas en distintos niveles del orden social para atenuar los graves efectos comentados con anterioridad ${ }^{1-5}$.

Como reflejo de las políticas para incrementar la calidad y la seguridad de la atención médica al paciente, en el Hospital Central Militar se han incrementado desde 2012 las acciones orientadas a optimizar los diversos procedimientos de atención médico-quirúrgica a las EO y UO. Aunado a ello, el interés de las altas autoridades de la institución para promover la competencia profesional y la reciente modernización en las instalaciones del departamento de urgencias han permitido atender de manera eficiente padecimientos cardiovasculares y coagulopatías, así como abordar a la paciente durante la expulsión del producto en un área especialmente designada para tal fin y con un protocolo especializado («Código binomio»). Creemos, por tanto, que todo lo anterior coadyuva potencialmente a incrementar la sobrevida en estos casos y a disminuir la incidencia y la prevalencia de complicaciones, sobre todo aquellas de naturaleza coagulopática, fenómenos tromboembólicos, accidentes neurológicos e insuficiencia orgánica. 
Este estudio exhibe la experiencia que haciendo uso de estos recursos humanos, materiales y administrativos ha ganado la institución, posicionándose como un hospital modelo en lo referente a la atención de pacientes críticos, incluyendo los casos de EO y UO.

Aun cuando la mayor parte de las admisiones al departamento de urgencias son casos de hemorragia posoperatoria categorizados con «Código rojo» en la escala de triage obstétrico $(n=6 ; 12.5 \%)$, es notable que el $83 \%(n=40)$ de los casos obstétricos atendidos en nuestro hospital no son EO. Sin embargo, varios de ellos, sin duda, en calidad de UO, representan una población que se beneficia de un sistema de atención oportuna, delineada por las escalas y los procedimientos de asignación de prioridad de la atención médica con que cuenta la institución (Tabla 2). La mayor parte de las UO son casos de amenaza de aborto y trastornos hipertensivos no graves del embarazo, condiciones clínicas que se prestan para la implementación de un abordaje diagnóstico y terapéutico por parte de los médicos especialistas, empleando con buenos resultados los protocolos correspondientes específicos para los padecimientos en cuestión que están establecidos en los manuales departamentales, y definiendo posteriormente su estancia hospitalaria o la referencia al hospital de especialidades gineco-obstétricas.

Por otro lado, la EO, tal como está definida operativamente en la institución, por fortuna se presentó en un porcentaje menor de casos $(17 \% ; n=8)^{1,2,6-8}$. En estas situaciones, para su adecuado abordaje, en el departamento de urgencias se activa el protocolo denominado "Código máter» $»^{1,2,4-7}$, que a su vez alerta, conforma y acciona a los elementos del EMRIO. Los diversos integrantes de este equipo proceden a la atención de la paciente según el procedimiento que sea aplicable al padecimiento específico con el cual esta arribe, solicitando por vía de regulaciones administrativas especiales la intervención de especialistas en gineco-obstetricia y pediatría pertenecientes al HMEMYN, que es una instalación cercana a nuestro hospital, los cuales arriban dentro de los siguientes 20 minutos a partir del llamado. Al estratificar la naturaleza de las indicaciones de activación del protocolo "Código máter» encontramos que las afecciones que con más frecuencia lo motivan son los casos de hemorragia posoperatoria y los trastornos hipertensivos graves del embarazo y sus complicaciones, lo cual hace posible que tras la evaluación cardiológica y neurológica adecuada, así como su tratamiento en las unidades de hospitalización regular o incluso de cuidados intensivos cardiovasculares, puedan ser referidas al centro de especialidad correspondiente con mejoría notable en sus condiciones físicas. Por mala fortuna, en estos casos en concreto también ha sido requerida la intervención de especialistas quirúrgicos, específicamente para el tratamiento de tres pacientes con rotura hepática por HELLP. Por su permanente disposición en nuestro hospital, en estas tres pacientes también fue posible practicar la angiografía con angioembolización selectiva como método adyuvante a la hepatostasia operatoria de las pacientes.

Representa un hecho notable para nuestra institución que en una paciente con puerperio quirúrgico inmediato y sangrado coagulopático exanguinante, trasladada del HMEMYN, se practicó una toracotomía en el departamento de urgencias con excelente resultado para la vida y la función neurológica, lo cual es sin duda un punto de referencia nacional para la práctica del procedimiento, pues los reportes de aplicación de este en el paro cardiaco no traumático son anecdóticos 8,9 . De la misma forma, refleja el gran potencial de los recursos humanos y materiales de nuestro hospital para la atención de pacientes obstétricas in extremis.

La tasa global de complicaciones encontrada en nuestra serie fue del $27 \%(n=13)$, siendo las más comunes la falla renal $(6 / 13 ; 46 \%)$, la sepsis de origen pulmonar y la coagulopatía $(5 / 13 ; 38.5 \%)$, condiciones específicas que han sido señaladas en la literatura como las que se presentan con más frecuencia en los casos de morbilidad ${ }^{10-14}$. Esto contrasta con lo reportado para los hospitales generales, donde la tasa global de morbilidad es del $6-21 \%$. Consideramos que este fenómeno se debe fundamentalmente a que varios pacientes referidos arriban en condiciones anatómicas y fisiológicas de alto índice de gravedad, presentando ya de hecho complicaciones que fueron computadas para nuestro análisis y definiendo un curso clínico tórpido, lo cual incrementó aún más la tasa de morbilidad.

La mortalidad global en nuestra serie fue del $6.2 \%$ (3/48). Una paciente puérpera con neumopatía intersticial idiopática en etapa fibrótica falleció en la unidad de hospitalización regular, y otra cursando también su puerperio sucumbió con tromboembolia pulmonar masiva en el departamento de urgencias, para una tasa de muerte fuera de la UMI del $4.1 \%$ (2/48). Sin embargo, para analizar con mayor profundidad este rubro es necesario enfatizar la gran proporción de pacientes que reunieron los criterios de admisión a la 
UMI (23/48; 48\%). Como se mencionó, la alta puntuación APACHE II con la cual arribaron estas pacientes, que alcanzó el orden de 19.4 en la escala correspondiente, se relacionó con una alta posibilidad predicha de muerte (35.5\%). Hazelgrove, et al. ${ }^{10}$, en un estudio multicéntrico de admisiones obstétricas a 14 unidades de cuidados intensivos $(N=210)$, que representa quizá la serie específica más grande reportada en la literatura, encontraron una mortalidad materna del $3.3 \%$, definiendo como causas principales de muerte el estado de choque de diversos orígenes y las enfermedades cardiovasculares. Es visible que, a pesar de la correlación estadística entre la puntuación APACHE II y la mortalidad predicha, la tasa global de muerte en la UMI fue menor que la esperada y que la tasa media que apunta la literatura mundial (15\%; rango:1-33\% $)^{10-14}$. Esto, adicionalmente al reducido tamaño de la muestra, puede reflejar la oportunidad en la atención proporcionada y protocolizada de estas pacientes desde el mismo departamento de urgencias. De la misma forma, en la UMI se cuenta con los recursos humanos y materiales necesarios (dentro de los cuales destaca la terapia de reemplazo renal lento continuo) que en conjunto son indispensables para no interrumpir la atención oportuna y de alta especialidad que demandan estas pacientes.

En virtud de no contar con posibilidad de seguimiento de los productos, estamos conscientes de que este estudio carece del poder necesario para analizar la evolución clínica del binomio, lo cual es un factor importante para determinar la oportunidad, la calidad y la seguridad de la atención médico-quirúrgica a la madre, pues los índices pronósticos del producto son un indiscutible reflejo de lo anteriormente señalado.

En conclusión, como consecuencia de la postura del Gobierno Federal y de las Dependencias Oficiales interesadas, así como de las autoridades militares, el Hospital Central Militar ha delineado y definido diversos procedimientos para abatir la morbilidad y la mortalidad maternas. Tenemos la certeza razonable de que la correcta práctica de estos procedimientos y la estrecha colaboración con el HMEMYN contribuirán a alcanzar los objetivos institucionales deseados, y a coadyuvar a cubrir las prioridades relativas a la salud materna estatuidas en la Política Nacional de Salud ${ }^{3}$.

\section{Agradecimientos}

Agradecemos por su colaboración estadística y coautoría en este estudio al Mayor M.C. Óscar Enrique Pérez-Morales y a la Mayor M.C. Gabriela Alhelí Guillén-Hernández.

\section{Conflicto de intereses}

Los autores declaran no tener conflictos de intereses.

\section{Bibliografía}

1. Subsecretaría de Prevención y Promoción de la Salud. Lineamiento Técnico 2016 "Triage Obstétrico, Código Máter y Equipo de Respuesta Inmediata Obstétrica". Ciudad de México (México): Secretaría de Salud; 2016. 60 p. (Consultado el 20 de diciembre de 2017.) Disponible en: https://www.gob.mx/cms/uploads/attachment/file/270472/web_TriageObstetricoCM.pdf

2. Salinas AA, Villegas JM. Guía técnica para el funcionamiento del Código Máter en las unidades hospitalarias de segundo nivel de atención médica del Instituto de Salud del Estado de México. Toluca (Estado de México): Instituto de Salud del Estado de México; 2009. 13 p. (Consultado el 21 de diciembre de 2017.) Disponible en: https://es.scribd.com/ doc/66530700/Guia-Tecnica-Para-El-Codigo-Mater-en-Unidades-Hospital-Arias

3. Subsecretaría de Prevención y Promoción de la Salud. Salud materna. Ciudad de México (México): Secretaría de Salud; 2015. 39 p. (Consultado el 21 de diciembre de 2017.) Disponible en: http://www.senado.gob.

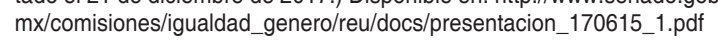

4. Diario Oficial de la Federación. Norma Oficial Mexicana NOM-007SSA2-2016 para la atención de la mujer durante el embarazo, parto y puerperio, y de la persona recién nacida. Ciudad de México (México): Secretaría de Gobernación; 2016. 22 p. (Consultado el 20 de diciembre de 2017.) Disponible en: http://www.dof.gob.mx/nota_detalle.php?codigo=5432289\&fecha=07/04/2016

5. Alvarado BRM, Arroyo VM, Hernández PC, Vélez CM, Márquez TP. Manejo del triage obstétrico y código máter en el Estado de México. Enfermería Universitaria. 2012;9:61-71.

6. Fescina R, De Mucio B, Ortiz El, Jarquin D. Guía para la atención de las principales emergencias obstétricas. Montevideo (Uruguay): Organización Panamericana de la Salud; 2012. 76 p. (Consultado el 20 de diciembre de 2017.) Disponible en: http://www.paho.org/clap/index. php?option=com_docman\&view=download \&category_slug=salud-de-mujer-reproductiva-materna-y-perinatal\&alias=279-guias-para-la-atencion-de-las-principales-emergencias-obstetricas-1\&Itemi$\mathrm{d}=219$ \&lang=es

7. Briones GJC, Gutiérrez VMC, Esquivel GLJ, de León PM, Briones VCG. Experiencia exitosa con el Código Máter. Med Crit Ter Int. 2011;25:43-5.

8. Committee on Obstetric Practice and the American Academy of Pediatrics' Council Environmental Health. Committee opinion no. 726: Hospital disaster preparedness for obstetricians and facilities providing maternity care. Obstet Gynecol. 2017;130:291-7.

9. Kornhall DK, Dolven T. Resuscitative thoracotomies and open chest cardiac compressions in non-traumatic cardiac arrest. World J Emerg Surg. 2014;9:54-9.

10. Hazelgrove JF, Price C, Pappachan VJ, Smith GB. Multicenter study of obstetric admissions to 14 intensive care units in Southern England. Crit Care Med. 2001;29:770-5.

11. Anwari JS, Butt AA, Al-Dar MA. Obstetric admissions to the intensive care unit. Saudi Med J. 2004;25:1394-9.

12. Okafor UV, Aniebue U. Admission pattern and outcome in critical care obstetric patients. Int J Obstet Anesth. 2004;13:164-6.

13. Hasbún HJ, Sepúlveda-Martínez A, Cornejo RR, Romero PC. Intensive care admissions due to severe maternal morbidity. Rev Med Chil. 2013;141:1512-9.

14. Thakur M, Gonik B, Gill N, Awonuga AO, Rocha FG, González JM. Intensive care admissions in pregnancy: analysis of a level of support scoring system. Matern Child Health J. 2016;20:106-13. 